\title{
Pandemic escape plan: Discontinuing policies that have reached their expiry date
}

The Latin phrase 'Primum non nocere', meaning 'First, do no harm', is a key principle in medicine. However, few interventions, whether at individual or community level, are without harmful effects. Practitioners therefore have to weigh up the potential benefits and negative effects of any medical or surgical intervention. The same applies to public health-level interventions for COVID-19.

Restricting human movement and limiting the size of gatherings have substantial public health benefit when appropriately applied. ${ }^{[1]}$ However, one should always carefully compare anticipated benefit v. harm. The World Health Organization defines health as a state of complete physical, mental and social wellbeing, and not merely the absence of disease or infirmity. ${ }^{[2]}$ As humans are social creatures, social isolation, especially of children and the elderly, carries a huge mental health cost. ${ }^{[3]}$ Similarly, COVID-19-associated disruptions of healthcare services, education, sports and leisure may all have harmful effects. ${ }^{[4,5]}$

A major reason why the COVID-19 pandemic has been devastating is that individuals who are immunologically naive and have other risk factors such as older age or medical comorbidities have a substantial risk of serious disease or death. ${ }^{[6]}$ However, this risk drops substantially after vaccination. ${ }^{[7]}$ Although the risk of infection increased with the emergence of variants of concern $(\mathrm{VoC})$ such as Delta and Omicron that display partial immune escape, vaccines remained valuable in reducing the risk of hospitalisation. ${ }^{[8,9]}$ Similarly, prior natural infection, although associated with an initial high risk of severe disease, reduces the subsequent risk of severe illness. ${ }^{[10,11]}$ Data emerging on the rate of infections and hospitalisation with the Omicron VoC highlight that prior exposure (either by vaccine or natural infection), although not protective against infection per se, offers substantial protection against hospitalisation and death. ${ }^{[12]}$ Coupled with a likely lower virulence of the Omicron VoC, ${ }^{[13]}$ this could explain the low number of deaths during the peak of the fourth wave of infections in South Africa.

Eradication of SARS-CoV-2 is not an achievable goal; the virus is almost certain to continue to circulate and evolve. ${ }^{[14]}$ The pattern of circulation is already shifting from pandemic to endemic in many regions of the world. The highly contagious Omicron variant makes continued avoidance of infection next to impossible; Omicron may well infect the vast majority of human beings over the foreseeable future. SARS-CoV-2 epidemiology is changing from an epidemic pattern to an endemic pattern, with primary infections in childhood, when the risk of severe disease is lower, followed by frequent reinfections over one's lifetime. ${ }^{[15,16]}$ This is the pattern seen with the four endemic human coronaviruses, each of which probably started with a pandemic similar to the present one. ${ }^{[15,17]}$ In individuals with prior immunity, and who are at low risk of severe COVID-19 disease, breakthrough infections (defined as infections in vaccinated individuals) or reinfections (infections in those with prior infection) are usually mild. ${ }^{[18]}$

Prevention of all SARS-CoV-2 infections is no longer an appropriate or achievable goal. Indeed, breakthrough infections may even be beneficial, offering broader immune protection than vaccines on their own. This 'super-immunity' is likely to be more durable and more protective against future emerging variants than any available vaccine, and infection would not carry a high risk of serious disease for those with vaccine-induced basic immunity. Whereas vaccination had limited effect in preventing transmission of the SARS-CoV-2 Delta variant, natural infection with the competing Omicron variant appears to induce high neutralising antibody titres, which may be why Omicron seems to be outcompeting Delta. ${ }^{[19]}$

Nevertheless, although breakthrough infection in previously vaccinated individuals might be beneficial, it could still cause serious disease in those who are poor vaccine responders. Emphasis must therefore be placed on protecting those who are least likely to be protected well through vaccination, rather than preventing SARS$\mathrm{CoV}-2$ transmission in the general population, which is neither feasible nor necessary.

As we enter the post-pandemic phase, the following principles should guide interventions providing optimal benefit while doing the least possible harm:

- The main and proven benefit of vaccination is for individual protection. Priority should be given to reaching remaining unvaccinated highrisk individuals (the elderly and those with other risk factors) and to providing additional (booster) doses where needed.

- Research on identifying and optimally managing groups at high risk of severe disease should continue, including infants and young children, and assessing the risk-benefit ratio of vaccination in each group.

- Simple and cheap measures with few or no disadvantages, such as mask wearing in congested indoor public spaces and good hand hygiene, should continue as they reduce the risk not only of COVID19 but other infections too.

- Protection of individuals not reliably protected by vaccination, such as haematopoietic stem cell transplantation recipients, should be based on reducing their exposure to SARS-CoV-2. Measures to reduce the risk of transmission by healthcare workers caring for these individuals could involve booster vaccination, symptom screening, frequent antigen-based testing, and the wearing of FFP2/ N95 respirators.

- Options for optimised, where possible and feasible, individualised management of high-risk individuals should be investigated; these could include determining antibody titres to guide vaccine booster doses, pre-exposure prophylaxis, early intervention with monoclonal antibodies or antiviral drugs, etc.

- Policies that require SARS-CoV-2 testing prior to hospital admission, for elective procedures, should be reconsidered and should rather emphasise vaccination and consider the risk of severe COVID-19 in vaccinated individuals v. the risk of complications of other respiratory infectious agents not routinely tested for (e.g. tuberculosis, influenza and rhinovirus).

- Quarantine should cease for most scenarios. Non-pharmaceutical measures such as wearing masks, distancing and testing can replace quarantine without unduly increasing risk. The exceptions are healthcare workers working with highly vulnerable individuals, e.g. immunocompromised patients and neonates. The duration of quarantine can possibly be adjusted according to immune status.

- With emerging SARS-CoV-2 immune escape variants, there is a lack of high-level evidence that vaccination would protect others. Policies that require vaccination of individuals for purposes other than an individual's own health should balance evidence of community benefit (not limited to medical issues) with individuals' rights.

Policies that were very important at the time to slow the rate of SARS-CoV-2 transmission and prevent healthcare services from becoming overwhelmed may soon reach their expiry date. The 
epidemiological situation has changed, as has the virus. Measures should be reconsidered as the risk of severe COVID-19 diminishes with high rates of immunity at population level. Policies need to keep up with emerging scientific data. Whereas mask wearing in poorly ventilated, crowded indoor spaces may maintain benefits, as it would protect against many other known or unknown infections, most other policies aimed at curbing the spread of COVID-19 might no longer be useful.

\section{Declaration. None.}

Acknowledgements. None.

Author contributions. GUvZ drafted the first version, AD and WP critiqued, commented on and edited it and added information and references. All authors approved the final version.

Funding. None.

Conflicts of interest. None.

\section{Gert U van Zyl}

Division of Medical Virology, Department of Pathology, Faculty of Medicine and Health Sciences, Stellenbosch University, Cape Town, South Africa; National Health Laboratory Service, Tygerberg Hospital, Cape Town, South Africa

guvz@sun.ac.za

\section{Angela Dramowski}

Division of Paediatric Infectious Diseases, Department of Paediatrics and Child Health, Faculty of Medicine and Health Sciences,

Stellenbosch University, Cape Town, South Africa

\section{Wolfgang Preiser}

Division of Medical Virology, Department of Pathology, Faculty of Medicine and Health Sciences, Stellenbosch University, Cape Town, South Africa; National Health Laboratory Service, Tygerberg Hospital, Cape Town, South Africa
1. Caristia S, Ferranti M, Skrami E, et al. Effect of national and local lockdowns on the control of COVID-19 pandemic: A rapid review. Epidemiol Prev 2020;44(5-6 Suppl 2):60-68. https://doi. org/10.19191/EP20.5-6.S2.104

2. World Health Organization. Constitution of the World Health Organization. https://www.who.int/ about/governance/constitution (accessed 6 January 2022).

3. Lewis K. COVID-19: Preliminary data on the impact of social distancing on loneliness and mental health. J Psychiatr Pract 2020;26(5):400-404. https://doi.org/10.1097/PRA.0000000000000488

4. United Nations Educational Scientific and Cultural Organization (UNESCO). Education: From disruption to recovery. 2020. https://en.unesco.org/covid19/educationresponse (accessed 8 January 2022).

5. Dunton GF, Do B, Wang SD. Early effects of the COVID-19 pandemic on physical activity and sedentary behavior in children living in the U.S. BMC Public Health 2020;20(1):1-13. https://doi. org/10.1186/s12889-020-09429-3

6. Jordan RE, Adab P, Cheng KK. Covid-19: Risk factors for severe disease and death. BMJ 2020;368:m1 198. https://doi.org/10.1136/bmj.m1198

7. Bubar KM, Reinholt K, Kissler SM, et al. Model-informed COVID-19 vaccine prioritization strategies by age and serostatus. Science 2021;371(6532):916-921. https://doi.org/10.1126/science.abe6959

8. Collie S, Champion J, Moultrie H, Bekker L-G, Gray G. Effectiveness of BNT162b2 vaccine against Omicron variant in South Africa. N Engl J Med 2021 (epub 29 December 2021). https://doi, org/10.1056/NEJMc2119270

9. Evans SJW, Jewell NP. Vaccine effectiveness studies in the field. N Engl J Med 2021;385(7):650-651. https://doi.org/10.1056/NEJMe2110605

10. Pulliam JRC, van Schalkwyk C, Govender N, et al. Increased risk of SARS-CoV-2 reinfection associated with emergence of the Omicron variant in South Africa. medRxiv 2021 (epub 2 December 2021). https://doi.org/10.1101/2021.11.11.21266068

11. Cele S, Jackson L, Khan K, et al. SARS-CoV-2 Omicron has extensive but incomplete escape of Pfizer BNT162b2 elicited neutralization and requires ACE2 for infection. medRxiv 2021 (epub 9 December 2021). https://doi.org/10.1101/2021.12.08.21267417

12. Maslo C, Friedland R, Toubkin M, Laubscher A, Akaloo T, Kama B. Characteristics and outcomes of hospitalized patients in South Africa during the COVID-19 Omicron wave compared with previous waves. JAMA 2021;30:e2124868. https://doi.org/10.1001/jama.2021.24868

13. McMahan K, Giffin V, Tostanoski LH, et al. Reduced pathogenicity of the SARS-CoV-2 Omicron variant in hamsters. bioRxiv 2022 (epub 3 January 2022). https://doi.org/10.1101/2022.01.02.474743

4. Eariant in ham Es. Osterholm M, Gounder CR. A national strategy for the 'new normal' of life with COVID. JAMA 2022;327(3):211-212. https://doi.org/10.1001/jama.2021.24282

15. Lavine JS, Bjornstad ON, Antia R. Immunological characteristics govern the transition of COVID-19 to endemicity. Science 2021;371(6530):741-745. https://doi.org/10.1126/science.abe6522

16. Antia R, Halloran ME. Transition to endemicity: Understanding COVID-19. Immunity 2021;54(10):2172-2176. https://doi.org/10.1016/j.immuni.2021.09.019

17. Corman VM, Muth D, Niemeyer D, Drosten C. Hosts and sources of endemic human coronaviruses. Adv Virus Res 2018;100:163-188. https://doi.org/10.1016/bs.aivir.2018.01.001

18. Lipsitch M, Krammer F, Regev-Yochay G, Lustig Y, Balicer RD. SARS-CoV-2 breakthrough infections in vaccinated individuals: Measurement, causes and impact. Nat Rev Immunol 2022;22(1):57-65. https://doi.org/10.1038/s41577-021-00662-4

19. Kitchin D, Richardson SI, van der Mescht MA, et al. Ad26.COV2.S breakthrough infections induce high titers of neutralizing antibodies against Omicron and other SARS-CoV-2 variants of concern. medRxiv 2022 (epub 4 January 2022). https://doi.org/10.1101/2021.11.08.21266049

S Afr Med J. Published online 27 January 2022. https://doi.org/10.7196/SAMJ.2022. v112i3.16381 